simply a matter of repeating the analyses and engineering of the past, because the grid is being transformed to accommodate significant amounts of power from highly variable sources, such as wind turbines and solar cells. These new sources, as they are currently controlled, also tend to contribute less to damping perturbations. Thus the transformation to a more sustainable energy system generally holds great promise, but also poses specific challenges for maintaining synchrony.
The grid is both a playground for physical and mathematical phenomena and a critical infrastructure that underpins our way of life. Recent advances in bridging the scientific and engineering realizations of network synchrony have already yielded some analytic conditions for synchrony, and the hope is that these studies can provide improvements to maintain grid stability, keep the lights on - and continue to optimize the brewing of coffee in the morning.
Ian Dobson is in the Department of Electrical and Computer Engineering, Iowa State University, 1117 Coover Hall, Ames, Iowa 50011, USA.

e-mail:dobson@iastate.edu

References

1. Motter, A. E., Myers, S. A., Anghel, M. \& Nishikawa, T. Nature Phys. 9, 191-197 (2013)

2. Strogatz, S. H. Physica D 143, 1-20 (2000)

3. Dörfler, F., Chertkov, M. \& Bullo, F. Proc. Natl Acad. Sci. USA http://dx.doi.org/10.1073/pnas.1212134110 (2013).

Published online: 10 February 2013

\title{
BIOMECHANICS
}

\section{Have legs will travel}

Centipedes happily scurry along in straight lines at low speeds. As soon as they pick up the pace, however, their motion is characterized by undulations (pictured) the amplitude and wavelength of which increase with speed. Common wisdom holds that these undulations impede centipede motion and meet resistance from muscles along the body axis. But there is some evidence to suggest that this muscular activity supports the undulations rather than resists them. A study by Shinya Aoi and co-workers has now revealed that both models are capable of giving rise to undulations, but do so using different mechanisms (Phys. Rev. E 87, 012717; 2013).

Aoi et al. investigated the dynamics of centipede locomotion using experiments featuring a centipede-like robot. Their mechanical model consisted of six modules connected by torsional springs, each comprising a rigid body flanked by a single pair of legs - a far cry from its multi-legged namesake, but the simplest mechanical structure capable of reproducing undulations. The robot's model nervous system reduced the task of controlling the legs to a series of simple periodic movements.

The researchers found that as stepping rate increased, lateral undulations appeared above a critical value. The speed of locomotion did not influence that of undulations, but instead had a pronounced effect on their amplitude and wavelength, as is the case for real centipedes.

Combining this result with a twodimensional minimal model and numerical simulations, Aoi et al. uncovered an instability suggestive of a supercritical Hopf bifurcation. The bifurcation was found to be determined largely by the relationship between spring stiffness, acting to stabilize a straight path, and floor friction, which emerged as a destabilizing factor. This behaviour is reminiscent of dynamics in other systems, including the jack-knife instability observed when a car pulls one or more trailers.

To test the competing theories, Aoi etal. implemented another robot model that traded the passive joints between modules for those with an active component. Whereas simple torsional springs were used to mimic muscular resistance in the passive model, the application of periodic torque to the joints lent an active contribution to this resistance. The active model produced similar undulations to those generated through the Hopf bifurcation, albeit through a different mechanism. 